\title{
Elementos de Design com Foco na Satisfação para Serious Games de Reabilitação e Condicionamento Físico Aplicados no jogo GiroJampa
}

\author{
Rebeca Raab Bias Ramos ${ }^{1}$, Liliane S. Machado ${ }^{1}$ \\ ${ }^{1}$ Universidade Federal da Paraíba (UFPB) \\ João Pessoa, Paraíba, Brasil \\ rebecabramosegmail.com, lilianeddi.ufpb.br
}

\begin{abstract}
Bearing in mind the importance of physical activities and knowing that serious games have been a stimulating alternative for their practice, this study aims to present new requirements for design with a focus on user satisfaction in serious games aimed at rehabilitation and physical conditioning. This proposition arose from the analysis of the development requirements of the GiroJampa game, aimed at people in wheelchairs.
\end{abstract}

Resumo. Tendo em vista a importância das atividades físicas e sabendo que os serious games tem sido uma alternativa estimulante para a prática das mesmas, o presente trabalho tem por objetivo apresentar novos requisitos para o design com foco na satisfação do usuário em serious games voltados à reabilitação e condicionamento físico. Esta proposição surgiu a partir da análise de requisitos de desenvolvimento do jogo GiroJampa, voltado a pessoas em cadeira de rodas.

\section{Introdução}

A deficiência em geral é um assunto que constantemente recebe mais destaque. Isso porque nos próximos anos a deficiência será uma preocupação ainda maior devido ao envelhecimento das populações, bem como ao aumento global de doenças crônicas tais como diabetes, doenças cardiovasculares, câncer e distúrbios mentais. As intervenções para superar as desvantagens associadas à deficiência são múltiplas, sistêmicas e complexas dependendo do grau de limitação do individuo [WHO $]^{1}$.

O uso de jogos como ferramentas no processo de estimulação motora e/ou cognitiva tem ganhado maior relevância nos últimos anos. Autores como [Dias et al. 2019] e [Kassee et al. 2017] destacam em seus trabalhos ganhos no desempenho ocupacional, função motora grossa, equilíbrio, força, nas atividades cotidianas, na função dos membros, na aderência ao tratamento e na motivação. Esta motivação está relacionada com a satisfação do usuário obtida com o uso do jogo. A satisfação, pode manter os jogadores interessados em continuar jogando, retornar a jogar e recomendar o jogo [O'Brien and Toms 2008]. Ela é uma medida obtida com a avaliação de jogos e objetiva conhecer os elementos que agradaram e motivaram o jogador. Assim sendo, é uma medição a posteriori.

Em 2021 foram apresentados requisitos de design com foco na obtenção de satisfação do usuário em serious games (SG) educacionais [Almeida and Machado 2021].

\footnotetext{
${ }^{1}$ https://apps.who.int/iris/bitstream/handle/10665/70670/WHO_NMH_VIP_11.01_por.pdf
} 
Tais requisitos buscaram complementar o processo de design de jogos a partir de uma visão específica com o intuito de potencializar os resultados esperados com o uso do jogo. Na presente pesquisa busca-se expandir estes requisitos que tem como foco a satisfação, propondo e discutindo novos requisitos e elementos que sejam específicos para SG em saúde voltados ao condicionamento físico e reabilitação de pessoas em cadeira de rodas.

\section{Objetivos e Contribuições}

Este trabalho tem como objetivo geral propor um conjunto de requisitos e elementos de design em SGs voltados para o condicionamento da saúde e reabilitação de pessoas em cadeira de rodas. Tais requisitos tiveram como foco específico a obtenção da satisfação do usuário (jogador). Para tanto, foram observados os aspectos relacionados à satisfação que poderiam ser considerados no desenvolvimento de um jogo intitulado GiroJampa.

Este objetivo foi particionado em três objetivos específicos a serem alcançados: (1) Analisar jogos existentes voltados à reabilitação; (2) Propor requisitos específicos, com foco na satisfação do usuário, para SGs voltados ao condicionamento físico e reabilitação de pessoas em cadeira de rodas; (3) Implementar um serious game aplicando os requisitos de design observados e propostos no ponto anterior.

\section{Metodologia}

O desenvolvimento deste trabalho foi dividido em três etapas, de modo a alcançar o objetivo geral e os objetivos específicos estabelecidos.

Etapa 1: Análise de trabalhos correlatos: Os artigos pesquisados [Lourenço 2018], [Batista et al. 2016], [Oliveira et al. 2013], e [Dias et al. 2019] destacam pontos importantes para desenvolvimento de SGs para reabilitação, tais como: ajustes automáticos de dificuldade para garantir que o jogador não perca a motivação, análise das pontuações que podem estar relacionadas também a motivação, uso de ferramentas não intrusivas na captação dos dados dos jogadores, análise de sessões anteriores do jogo para observação da progressão do jogador, movimentos com finalidade para cada tipo de deficiência, e questionário para medição de satisfação.

Etapa 2: Propor requisitos específicos, com foco na satisfação do usuário, para SGs voltados ao condicionamento físico e reabilitação de pessoas em cadeira de rodas: O trabalho de [Almeida and Machado 2021] divide os requisitos em nove categorias, sempre com foco na obtenção da satisfação. São estas: Concentração, Game Challenge, Habilidades do jogador, Controle, Clareza de metas, Feedback, Imersão, Interação Social e Conteúdo Educacional. Entretanto, a categoria de Conteúdo Educacional, não se aplica ao presente trabalho e por isso foi substituída pela categoria denominada de Conteúdo de Condicionamento de Saúde a fim de abordar jogos deste conteúdo especifico.

1. Requisitos de Concentração afetam os elementos relacionados à atratividade do jogo e atenção do jogador.

2. Requisitos do Game Challenge afetam os elementos relacionados à dificuldade do jogo e como essa se comporta com cada jogador.

3. Requisitos da Habilidade do jogador afetam os elementos relacionados à habituação do jogador à mecânica do jogo,interfaces e tecnologias. 
4. Requisitos de Controle afetam elementos relacionados à capacidade de resposta do jogo às entradas do jogador e elementos relacionados a como o jogo se comporta de acordo às ações realizadas pelo jogador.

5. Requisitos de Clareza de Metas afetam os elementos relacionados à apresentação dos objetivos do jogo e de aprendizagem para o jogador.

6. Requisitos de Feedback afetam elementos informativos sobre questões importantes que o jogador precisa para estar ciente.

7. Requisitos de Imersão afetam os elementos que fazem elevar o mundo do jogo e encoraja o jogador a sentir parte disso.

8. Requisitos de Interação Social afetam os elementos responsáveis pela comunicação e interação entre jogadores.

9. Requisitos de Conteúdo de Condicionamento de Saúde afetam elementos relacionados às capacidades físicas do jogador e sua evolução durante o processo avaliativo de esforço durante o jogo.

Devido à carência de requisitos que tivessem relação com a satisfação do usuário nesta categoria de SG, foram elaborados três requisitos que englobassem os pontos importantes relatados na etapa anterior. São requisitos propostos nesta categoria: 1. O jogo deve oferecer escala para avaliação de esforço, 2. O jogo deve oferecer exposição da evolução física do jogador, e 3. O jogo deve oferecer recompensas pela evolução física do jogador. Considerando a nova categoria de requisitos, foram propostos também elementos a serem utilizados nos SGs.

Etapa 3: Implementação dos elementos propostos em um SG voltado à reabilitação. O SG GiroJampa foi proposto para auxiliar no processo de reabilitação e condicionamento físico de pessoas em cadeira de rodas. Neste processo de design foram incluídos elementos no jogo de acordo com sua abordagem. Adicionalmente, foram implementados elementos da categoria Conteúdo de Condicionamento de Saúde. Estes se encontram no GiroJampa da seguinte forma:

1. O jogo deve oferecer escala para avaliação de esforço. Onde, foi utilizada a escala de BORG [Cabral et al. 2020] e foi implementada uma tela interativa na qual o jogador pode informar qual o Esforço Percebido após a realização de cada atividade;

2. O jogo deve oferecer exposição da evolução física do jogador. Abordado a partir da implementação de uma Tela de Desempenho e Progresso para o jogador;

3. O jogo deve oferecer recompensas pela evolução física do jogador. Foram implementados dois elementos na forma de Tela de troféus e Quadro de medalhas, recompensando o jogador ao longo de sua evolução.

\section{Resultados Obtidos}

Ao considerar SGs para reabilitação e condicionamento físico, este trabalho altera a proposta dos autores [Almeida and Machado 2021], de modo a substituir os requisitos de Conteúdo Educacional por requisitos de Condicionamento da Saúde e propor elementos nesta nova categoria. Além dessa nova categoria, foi necessário adicionar novos requisitos na categoria de Controle, para atender a parte de sensorização do jogo que considera a entrada das ações do usuários a partir de dispositivos capazes de capturar os dados do exercício físico realizado no jogo. Este conjunto de requisitos pode ser observado na Tabela 1, já com a adição da modificação proposta. 
Para a categoria proposta neste trabalho de conteúdo de condicionamento de saúde. Justifica-se os requisitos:

O jogo deve oferecer escala para avaliação de esforço: Por exigir esforço físico do paciente/jogador, é necessário que o jogo ofereça uma forma de medição deste. Para isso, são utilizadas escalas clínicas de avaliação do esforço para adaptação dos exercícios ao nível condicional de cada individuo.

O jogo deve oferecer exposição da evolução física do jogador: Permitindo que o jogador acompanhe seu desempenho, identifique seus pontos fortes e fracos, além de poder corrigir seus erros ao longo do jogo.

O jogo deve oferecer recompensas pela evolução física do jogador: A fim de aumentar a satisfação dos jogadores e os estimularem a voltarem a jogar.

Para a categoria de Controle foram adicionados os seguintes requisitos: (1) O jogo deve ter sensores que realizam a captura de informações sobre o jogador; (2) Os sensores do jogo devem atuar de modo não intrusivo, ou seja, não atrapalhando os movimentos dos jogadores; (3) Os aparelhos de medição do jogo podem ser ajustados pelo jogador. Estes requisitos buscam oferecer um feedback tátil e foram implementados a partir da sensorização de um ergômetro, plataforma sugerida por Souto et al. [Souto E.C. 2019].

Na Figura 1 é possível observar como os elementos destrinchados na terceira coluna na Tabela 1 estão implementados no GiroJampa. Além desses, foram propostos no total, quinze elementos para compor o jogo.

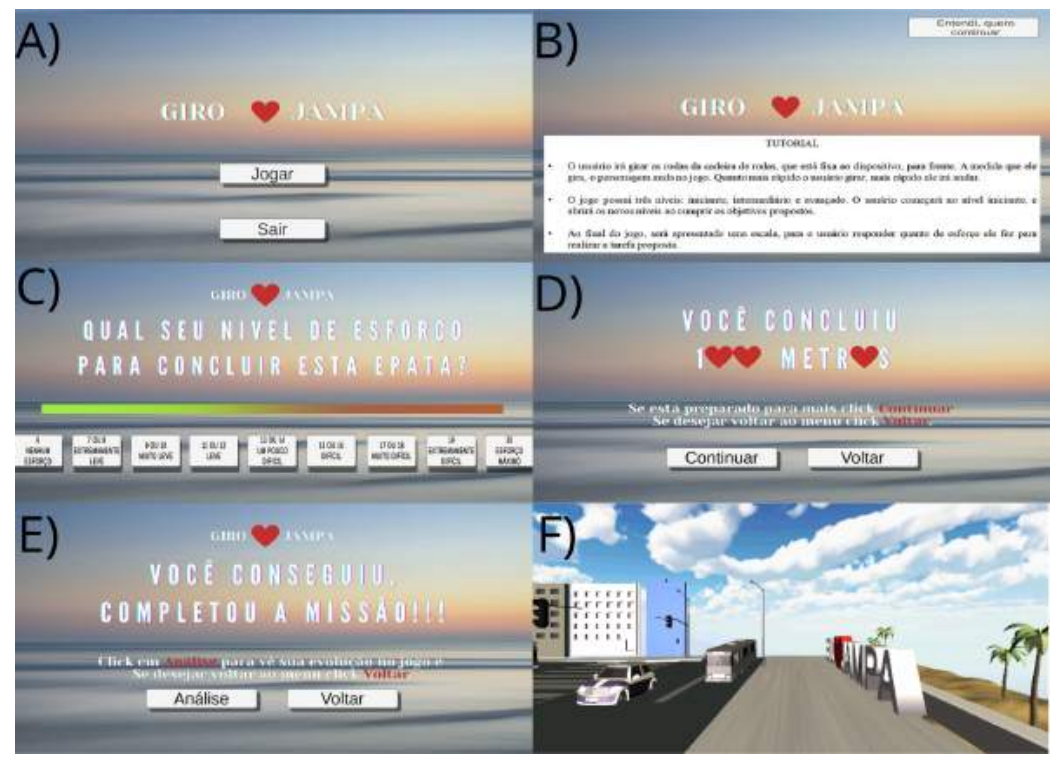

Figura 1. Algumas telas do GiroJampa: A) tela inicial; B) tutorial; C) solicitação do esforço percebido; D) informação da distância percorrida ao final de uma etapa; E) conclusão da missão; F) cenário na visão do jogador.

Vale ressaltar que as necessidades relacionadas ao condicionamento físico que levaram à definição dos requisitos foram definidas por uma equipe multidisciplinar. Até o momento, os elementos marcados em verde na Tabela 1 estão implementados e funcionais no GiroJampa. Os elementos em vermelho estão sendo implementados. 
Tabela 1. Requisitos de Design do Jogo GiroJampa.

\begin{tabular}{|c|c|c|}
\hline Requisitos de Design & Elementos sugeridos usados no jogo & Elementos no jogo \\
\hline \multicolumn{3}{|l|}{ Concentração } \\
\hline $\begin{array}{l}\text { Cc1 O jogo deve ter elementos que chamem a atenção do jogador. } \\
\mathrm{Cc} 3 \text { O jogo deve ser capaz de prender a atenção do jogador enquanto ele executa as tarefas } \\
\mathrm{Cc} 6 \text { Os elementos do jogo não devem distrair o jogador das tarefas nas quais ele deseja se } \\
\text { concentrar. }\end{array}$ & Animaçôes & $\begin{array}{l}\text { 1. Cenário } \\
\text { 2. Animação responsiva }\end{array}$ \\
\hline $\begin{array}{l}\text { Cc2 O jogo deve ter estímulos que não impeçam o jogador de atingir os objetivos do jogo. } \\
\mathrm{Cc5} \mathrm{A} \text { carga de trabalho do jogo não deve exceder os limites esperados do público-alvo. } \\
\mathrm{Cc7} \text { Os elementos no jogo não devem distrair o jogador das tarefas nas quais ele precisa se } \\
\text { concentrar. }\end{array}$ & Telas informativas & $\begin{array}{l}\text { 3. Tutorial } \\
\text { 4. Tela informativa de distância } \\
\text { percorrida } \\
\text { 5. Tela informativa de tempo } \\
\text { 6. Tela interativa para Esforço } \\
\text { Percebido de Borg } \\
\end{array}$ \\
\hline \multicolumn{3}{|l|}{ Desafio de jogo } \\
\hline $\begin{array}{l}\text { Gc1 Os desafios do jogo devem corresponder ao nivel de habilidade esperado da base de } \\
\text { jogadores alvo. } \\
\text { Gc2 Os desafios do jogo devem se adaptar ao nivel de habilidade de cada jogador. } \\
\text { Gc4 O jogo deve apresentar novos desafios em um ritmo apropriado. }\end{array}$ & Dificuldade adaptativa & $\begin{array}{l}\text { 7. Avaliação do desempenho do } \\
\text { usuário } \\
\text { 8. Balanceamento do nivel de } \\
\text { dificuldade }\end{array}$ \\
\hline \multicolumn{3}{|l|}{ Habilidades do jogador } \\
\hline $\begin{array}{l}\text { Ps2 O jogo deve apresentar maneiras de permitir que o jogador aprenda o jogo que seja parte } \\
\text { da diversão esperada. }\end{array}$ & Conquistas & 9. Tela de troféus \\
\hline $\begin{array}{l}\text { Ps3 O jogo deve fornecer suporte para o jogador superar as dificuldades. } \\
\text { Ps4 O jogo deve permitir que o jogador aprenda a jogar por meio de um tutorial ou em um } \\
\text { estágio inicial. }\end{array}$ & Tutorial & 3. Tela de Tutorial \\
\hline $\begin{array}{l}\text { Ps5 O jogo deve fornecer ao jogador tempo e espaço para melhorar suas habilidades à } \\
\text { medida que progride Sistema de progressão,através do jogo para superar os desafios } \\
\text { subsequentes. }\end{array}$ & Desbloqueio gradual de recursos & $\begin{array}{l}\text { 7. Avaliação do desempenho do } \\
\text { usuário } \\
\text { 8. Balanceamento do nivel de } \\
\text { dificuldade }\end{array}$ \\
\hline Ps6 O jogo deve recompensar os jogadores por seus esforços. & Recompensas & 10. Tela de Troféus \\
\hline Ps7 A interface do jogo deve ser fácil de aprender e usar. & Tela de ajuda facilmente acessível & \\
\hline Ps8 A mecânica do jogo deve ser fácil de aprender & Modo de treinamento & 3. Tela de Tutorial \\
\hline \multicolumn{3}{|l|}{ Controle } \\
\hline $\begin{array}{l}\text { Ct3 O jogo deve permitir que o jogador pause ou pare o jogo para retomar o jogo mais tarde } \\
\text { Sistema de pausa, sem perder seu progresso. }\end{array}$ & $\begin{array}{l}\text { Sistema de pausa } \\
\text { Salve e carregue o sistema }\end{array}$ & 11. Botão de pausar. \\
\hline $\mathrm{Ct} 4 \mathrm{O}$ jogo não deve permitir que o jogador cometa erros que o impeça de progredir. & Feedback tátil & $\begin{array}{l}\text { 7. Avaliação do desempenho do } \\
\text { usuário }\end{array}$ \\
\hline Ct5 O jogo deve permitir que o jogador sinta que suas ações têm impacto no mundo do jogo. & $\begin{array}{l}\text { Reconhecimento do personagem do } \\
\text { jogador }\end{array}$ & 13. Tela de Desen \\
\hline \begin{tabular}{|l}
$\mathrm{Ct} 6 \mathrm{O}$ jogo deve ter sensores que realizam a captura de informações sobre o jogador. \\
\end{tabular} & Feedback tátil & 14. Sensorização do ergômetro \\
\hline $\begin{array}{l}\text { Ct7 Os sensores do jogo devem atuar de modo não intrusivo, ou seja, não atrapalhando os } \\
\text { movimentos dos jogadores. }\end{array}$ & Feedback tátil & \\
\hline Ct9 Os aparelhos de medição do jogo podem ser ajustados pelo jogador. & Feedback tátil & \\
\hline Ct10 O jogo deve oferecer suporte para entrada de dados do jogador vinda dos sensores. & Animações fluídas & \\
\hline \multicolumn{3}{|l|}{ Clareza de Metas } \\
\hline Cg1 O jogo não deve conter momentos em que o jogador não saiba o que fazer a seguir. & Tela de ajuda facilmente acessivel & 3. Tela de Tutorial \\
\hline $\begin{array}{l}\text { Cg2 Os objetivos principais do jogo devem ser apresentados ao jogador no momento } \\
\text { apropriado. }\end{array}$ & Log de progresso & \\
\hline Cg3 Os objetivos principais do jogo devem ser apresentados de forma clara. & Registro objetivo & \\
\hline $\begin{array}{l}\text { Cg6 O jogo deve deixar claro quais são os objetivos de aprendizagem para seu conteúdo } \\
\text { especifico. }\end{array}$ & Avaliação de desempenho & 15. Tela de Objetivos \\
\hline \multicolumn{3}{|l|}{ Feedback } \\
\hline Fb1 O jogo deve fornecer feedback ao jogador sobre seu progresso. & Log de progresso & 13. Tela de Desempenho e Progress \\
\hline $\mathrm{Fb} 2 \mathrm{O}$ jogo deve responder às ações do jogador. & Interações responsivas & $\begin{array}{l}\text { 7. Avaliação do desempenho do } \\
\text { usuário } \\
\text { 8. Balanceamento do nivel de } \\
\text { dificuldade }\end{array}$ \\
\hline Fb3 O jogo deve permitir que o jogador acesse sua pontuação sempre que achar necessário. & Classificação e pontuação do jogador & \\
\hline Fb4 O jogo deve deixar claro quais eventos ocorridos são importantes. & Cenas relacionadas ao progresso & \\
\hline Fb5 O jogo deve informar o jogador sobre o fracasso de sucesso de forma adequada. & Telas de vitória e derrota & 13. Tela de Desempenho e Progre \\
\hline \multicolumn{3}{|l|}{ Imersão } \\
\hline Im1 O jogo deve permitir que o jogador se sinta parte do mundo do jogo. & Animações e efeitos visuais & \\
\hline $\operatorname{Im} 2 \mathrm{O}$ jogo deve fornecer um mundo no qual o jogador possa se envolver emocionalmente. & Animações e efeitos visuais & 1. Cenário \\
\hline Im3 O jogo deve ser capaz de afetar as emoções e o humor do jogador. & Animações e efeitos visuais & 2. Animação responsiva \\
\hline \multicolumn{3}{|l|}{ Conteúdo de condicionamento de saúde } \\
\hline Hcc1 O jogo deve oferecer escala para avaliação de esforço & $\begin{array}{l}\text { Sistema de progressão } \\
\text { ou } \\
\text { Seleção de dificuldade }\end{array}$ & $\begin{array}{l}\text { 6. Tela interativa para Esforço } \\
\text { Percebido de Borg }\end{array}$ \\
\hline Hcc2 O jogo deve oferecer exposição da evolução física do jogador. & Log de progresso & 13. Tela de Desempenho e Progre \\
\hline Hcc3 O jogo deve oferecer recompensas pela evolução fisica do jogador & Recompensas & $\begin{array}{l}\text { 9. Tela de Troféus } \\
\text { 10. Quadro de medalhas }\end{array}$ \\
\hline
\end{tabular}

\section{Considerações finais e próximos passos}

Este trabalho propõe novos requisitos para o design com foco na obtenção da satisfação do jogador para SGs voltados à reabilitação e condicionamento físico. Tais requisitos visam auxiliar o desenvolvimento de tais SGs de modo que estes sejam capazes de propiciar 
maior satisfação aos potenciais usuários e, consequentemente, melhores resultados considerando a finalidade do SG. Os requisitos propostos e alterações ao modelo proposto previamente [Almeida and Machado 2021] foram aplicados no design do SG GiroJampa.

Como próximos passos desta pesquisa, planeja-se finalizar a implementação do GiroJampa e realizar testes de satisfação com potenciais usuários a fim de constatar a eficácia dos elementos implementados na satisfação do jogadores. Para tanto, espera-se utilizar o questionário GameFlow [Felix et al. 2018], específico para avaliar a satisfação em jogos.

\section{Referências}

Almeida, J. L. F. and Machado, L. (2021). Design requirements for educational serious games with focus on player enjoyment. Entertainment Computing, 38:100413.

Batista, T. V., Machado, L. S., and Valença, A. M. G. (2016). Surface electromyography for game-based hand motor rehabilitation. In 2016 XVIII Symposium on Virtual and Augmented Reality (SVR), pages 140-144. IEEE.

Cabral, L. L., Nakamura, F. Y., Stefanello, J. M., Pessoa, L. C., Smirmaul, B. P., and Pereira, G. (2020). Initial validity and reliability of the portuguese borg rating of perceived exertion 6-20 scale. Measurement in Physical Education and Exercise Science, 24(2):103-114.

Dias, T. d. S., Conceição, K. F., Oliveira, A. I. A., and Silva, R. L. M. (2019). Contribuições da gameterapia para as habilidades cognitivas de um adolescente com paralisia cerebral. Cadernos Brasileiros de Terapia Ocupacional, 27(4):898-906.

Felix, Z. C., Machado, L., Silva, J. R. O., Silva, A., and Almeida, L. (2018). Avaliando satisfação do usuário a partir dos modelos gameflow e pens: Um estudo com o jogo caixa de pandora mobile. Proceedings of SBGames.

Kassee, C., Hunt, C., Holmes, M. W., and Lloyd, M. (2017). Home-based nintendo wii training to improve upper-limb function in children ages 7 to 12 with spastic hemiplegic cerebral palsy. Journal of pediatric rehabilitation medicine, 10(2):145-154.

Lourenço, J. M. B. (2018). Jogos sérios para reabilitação motora com realidade virtual. tese [mestrado em engenharia eletrotécnica e de computadores]. Master's thesis, Faculdade de Ciências e Tecnologia, Universidade de Coimbra.

O'Brien, H. L. and Toms, E. G. (2008). What is user engagement? a conceptual framework for defining user engagement with technology. Journal of the American society for Information Science and Technology, 59(6):938-955.

Oliveira, D. M., Maciel, A. B. d. R., Carneiro, M. I. S., Cardoso, A. C. d. A., Gama, A. E. F. d., Chaves, T. d. M., Teichrieb, V., Araújo, C. C. d., and Monte-Silva, K. K. (2013). Desenvolvimento e aprimoramento de um sistema computacional-ikapp-de suporte a reabilitação motora. Motriz: Revista de Educação Física, 19(2):346-357.

Souto E.C., Siebra C.A. and, S. L. (2019). Avaliação de um sistema de captura e visualização de parâmetros físicos em um ergômetro para cadeirantes. In XI Congresso Brasileiro de Atividade Motora Monitorada. Alagoas, Brasil. 